\title{
Analysis of Features and Singing Skills of Chinese Classical Poetry Songs
}

\author{
Fangheng Wang ${ }^{1, a}$ \\ ${ }^{1}$ Neijiang Normal University, Neijiang, 641000, China \\ aemail
}

Keywords: Chinese classical poetry songs, Artistic characteristics, Singing skills

\begin{abstract}
Chinese classical poetry songs are not only an important part of traditional Chinese poetry art, but also the treasure of our national vocal music art. This paper analyzes the characteristics of conception beauty of literature, melody beauty of rhythms, artistic beauty of tunes of Chinese classical poetry songs. This paper also gives the singing skills of Chinese classical poetry songs to provide some references for the relevant researchers.
\end{abstract}

\section{Introduction}

Classical poetry songs refer to the vocal music works which select the ancient Chinese classical poetry as lyrics. They are composed by the modern musicians, which are accompanied by artistic accompaniment instruments and accompaniment and performed in concert chamber music. It has unique artistic charm and aesthetic features. Classical poetry songs in ancient times are a kind of poetry and singing with the music genre. It is the traditional music in some literati poetry music lyrics, mostly from the ancient literati poetry masterpiece. As a result of the existence and development of literati music, many poems and songs of the literati in the past dynasties were brought into the music. These ancient poems were consistent with the rhythm of the music. They became the Chinese classical poetry songs with lofty artistic conception and special charm. The Chinese classical poetry songs are a whole concept, which is a special vocal music genre Chinese art songs. They are different from both ancient and classical poems handed down and wrote for the lyrics, but also different from the modern poetry art songs from the May 4 Patriotic Movement. Chinese classical poetry songs are a vocal music genre China classical poetry combined with music, is a shining pearl China literary history, thousands of years of being widely praised. The most prominent feature of classical poetry and songs is that the lyrics come from classical poetry. With its refined language, lofty artistic conception and profound connotation, it conveys the unique charm of Chinese national culture. Through poetry, music and voice to express thoughts and feelings, Chinese classical poetry songs not only have thousands of years of traditional music culture, but also have the infiltration of modern multicultural for centuries with distinctive national style and times.

\section{Artistic Characteristics of Chinese Classical Poetry Songs Artistic Beauty}

Conception Beauty of Literature. Literature is the premise of music creation and enriches music inspiration, while music deepens literature and explores the inner artistic conception of literature. The Chinese classical poetry song is a perfect combination of music and poetry. It not only embodies the characteristics of Chinese traditional folk music, especially in the rhythm of the music, but also reflects the artistic charm of the artistic conception of literature. The origin and development of the Chinese classical poetry songs can be traced in the music form of preqin Dynasty poem dancing and the saying of "poetry for the heart and sound for the body" in Han Dynasty, Tang and Song Poetry in poetry in the poetry, Chinese itself to have the beauty of music rhythm. Ancient poetry is a poetic language, has high artistic conception of classical implicit prosody, vivid words, distinctive tone change ingenious structure and. Ancient poetry by indirect lyrical ways such as lyric, depict the fascinating realm of poetry, words to express feelings of his brilliant records. The special language forms of ancient poems form the classical poetry songs, and their lyrics are beautiful and rhythmic. 
Chinese classical poetry and artistic songs of the tune and lyrics of exquisite origin are the masterpiece of Chinese literati. They are also worthy of the name of traditional Chinese art songs. Similar to the traditional artistic songs in the western countries, Chinese art songs. Chinese classical poetry art songs in western theory pay attention to the combination of traditional culture at the same time. way of expression and Chinese national style and national temperament and national feelings of the combination retains the characteristics itself, including the absorption of folk tunes, traditional opera elements. Chinese classical poetry art songs and foreign art songs are derived from the literary works of poetry. The songs of Chinese classical poetry are concise. The artistic conception is far-reaching expressing deep feeling.

Melody Beauty of Rhythms. Chinese characters are full of rhyme and beauty. Chinese poetry songs are endowed with their unique rhythm and beauty. Prosody, on the one hand, refers to the rhyme of poetry; on the other hand, the internal melody that runs through the whole text. The text of the tonal rhyme arrangement, the level of scattered, a light weight, form a beautiful and harmonious tone, give people a pleasant feeling. The origin of metrical consciousness in Chinese poetry is closely related to ancient music. The use of different rhythm tone has rules more and alternatively, constitute a statement cadence. In his poems, different level and oblique tones text staggered configuration can make the sentence tone rich and diverse, form a kind of musical beauty. Tone is one of the elements of Chinese phonetics and has the function of distinguishing the meanings of words. The Chinese have a tone, make Chinese have a melodious music of the United states. Although China traditional music itself in the penetration and influence of diversified culture, melody shows a variety of features, but based on the Western musical techniques as a means of the more prominent style and aesthetic China traditional music of classical poetry songs. The unique artistic charm of classical poetry songs has been steadily improved and strengthened. Chinese classical poetry is not only rich in content, but also structured and strictly restricted by rules. In terms of music, in order to follow this rhythm, Classical poetry songs adopt the musical structure rigorously and flexible, give full play to the rhythm of music between Chinese traditional folk music lyrics and reflect the diversity of classical poetry songs melody.

Artistic Beauty of Tunes. The vocal beauty of Chinese traditional music reflects the harmonic cavity essence charm of Chinese classical poetry songs. Sing the words during the solid cavity when the vowel is full, radio, clear, and cannot be used indiscriminately mixed cavity, according to the content of tone to master the rhythm. Sing a long cavity to the attention of the ups and downs tactfully, which cannot sing slightly because of its long straight and short cavity. The traditional vocal music aesthetic principle in China is in accordance with the characteristics of Chinese phonetics. It follows the characters of accurate and clear reading, smooth and melodious voice, melodious melody, delicate and sincere expression, and full-bodied style. The singing features of classical poems and songs are formed on the basis of the characteristics of Chinese language, and have unique charm. Chinese traditional vocal music vocal beauty, meaning beauty, reflects the Chinese classical poetry song harmonic cavity essence charm. Sing the words during the solid cavity when the vowel is full, radio, clear, and cannot be used indiscriminately mixed cavity, according to the content of tone to master the rhythm, not whack. Sing a long cavity to the attention of the ups and downs tactfully, cannot sing because of its long straight and short cavity to procrastination sing strong toughness, not dry and dull. The traditional vocal music aesthetic principle in China is in accordance with the characteristics of Chinese phonetics. It follows the characters of accurate and clear reading, smooth and melodious voice, melodious melody, delicate and sincere expression, and full-bodied style. The vocal performance of classical poetry and songs is basically similar to the singing requirements of traditional art songs in Europe. It also emphasizes the expression of sound and emotion as well as the control of breath and sound. The Chinese classical poetry songs have little emotional ups and downs of relatively large theme, so the singers should control the feeling, perform introvertedly to express the deep feelings by the melodious poetry connotation. 


\section{Singing Skills of Chinese Classical Poetry Songs}

Lead the Sound by the Singer's Breath. Breath is the foundation of singing. In the singing of Chinese classical poetry songs, we should pay more attention to the singer's breath. Chinese Classical poetry songs, words, sentence bearing rare melody long. They are similar to the Chinese drama inside. The classical poetry songs often use drag, a dozen or dozens of words consisting of the whole song lyrics, which is the form of the one word with multi tone. Therefore, the time value of each word is not too short in singing, generally accounted for three to five beats, or even longer. This is also the meaning of expressing the profound meaning of traditional poetry songs. But with good breath support, you can perfectly sing classical poetry, art songs. In singing, the intensity and length of the breath are also important. From this we can see that the good control of breath is especially important. According to the connotation of the expression of classical poetry art songs of the content, the poetry of the introduction, accurate and timely adjust breath, an important technical link in this song on excellence. Not with a good breath support ability, you can sing the best classical poetry songs. For the song singing skills have a description of the main characters, strength. Not every sound of each song is sung with great intensity, but it is necessary to adjust the breath accurately and flexibly according to the content conveyed by classical poetry songs and the emotion expressed. According to the needs of songs, the importance of the important technical links of the singing and dancing of the breath can be achieved better, so that the unique charm of classical poetry songs can be achieved. Breathing is a very important part of singing, and it is essential to express emotion and support the singing atmosphere. Classical poetry, songs, music and lyrics are different from other vocal music genres. Therefore, breathing becomes the key to express emotion and expression in Chinses ancient poetry songs.

Select the Tunes according to the Words of Poetry Songs. Language is the foundation of singing. All singing techniques should be trained and applied around the center of the language. They are the important accomplishments of classical poetry art songs, including reflecting the content of ideological and emotional lyrics and master the basic skills of pronunciation in the singing of the Chinse classical poetry art songs. The articulation to a proper extent accurately is a classical poetry and songs, which shows the culture under the national tradition, and its aesthetic standards are based on the national language of our country. All singing techniques should be trained and applied around the center of the language. Master the basic skills of articulation, is conducive to the creation of the language function, the expression of the ideological content of work, improve the art of singing performance. Classical poems and songs in articulation requires clear and accurate pronunciation, enunciation, and close to pay attention to pay attention to maintain good sound, cavity vowel before a word is not accurate, at the end of the song, the mouth and throat position not down before and not stable, to achieve rounded nature and position of unity. Articulation is an important link of singing, vocal music is a combination of language and music art, singing in the music to express feelings, tell a story. If the audience cannot hear clearly and cannot understand the singer's singing content, the vocal music will lose its unique artistic charm. Therefore, in accordance with the requirements of the western art songs articulation to the interpretation of China classical poetry art song is not desirable. The lyrics of classical poems and songs are composed of rhythmic classical poems, and their charm is unique. The singer in the interpretation of the work should first understand the work style and features, and to understand the characteristics of melody style cavity. Therefore, exquisite charm becomes the important symbol of classical poetry and songs distinguished from the general vocal music genre. The artistic function of the cavity is mainly embodied in the main aspects of the display charm, reflecting the singer's interpretation of the song.

Sing Wonderfully with a Silver Voice and Deep Feeling. The lyrics of the Chinese classical poetry songs have high cultural values. A long history and unique charm of classical poetry means that the music element of art connotation and the interpretation of the artistic conception in classical poetry songs plays an important role in the classical poetry songs melody. It is based on the characteristics of ancient poetry and classical poetry in Chinese history. It is a gorgeous flower. The content is implicit and the structure is neat. The conception is artistic and the style is created. On the 
basis of traditional music culture, so the whole music image and emotion in place or not directly related to the classical poetry and classical poetry songs artistic value and unique charm, related to China's traditional culture broad and profound spirit. The perfect combination of ancient poetry and the combination of "Pearl" and "Bi" in music makes the singing value of this special vocal form seem particularly important. In vocal music singing, the technique is for the emotion service, pays attention to uses the technique to express the emotion. This is for the unique charm of classical poetry songs, the same vocal skills have been secondary to the rich emotional expression in place, only the expression of emotion in place, to express the special charm of the classical poetry songs. In the singing of the use of sound, should make the voice lyric, moving, relaxation degree. We should avoid large and strong volume changes as contrast, should be mainly lyrical. Excessive volume changes will destroy the original artistic conception of classical poetry and art songs, so we should pay attention to the intensity of breath control, and pay attention to the continuity of breath. They will make the sound lost too much, too fast or too slow, including the resonant cavity space to remain open and moderate, excessive open throat and other heavy, nasal cavity and throat. It may cause damage to the work style and artistic conception. In the stage performance, singers should follow the historical origins and special programs of classical poetry songs, and show the performing patterns in line with them.

\section{Conclusion}

Chinese classical poetry songs are gems and the essence of Chinese culture. To display a Chinese classical poetry song thoroughly, we need to analyze them comprehensively. The singer needs to flexibly use these above skills to make the singing a kind of natural revelation from the inside to the outside, so that the music works can be interpreted perfectly.

\section{References}

[1] Jia Bing, From Duet to Song Cycle and to Rhyme Shifts - On the Relationship between the Way of Singing and the Perspective Change in Ancient [J]. Journal of Hengshui University, 2016, 18(2): 126-128.

[2] Liu Honghong, On the Popular Songs Reference to the Classical Poetry [J]. Journal of Langfang Teachers College (Social Sciences Edition), 2011, 27(1): 57-59.

[3] Cen Zeli, Analysis of Classical Poetry’s Flavors in Contemporary Pop Songs [J]. Journal of Guangzhou Open University, 2012, 12(4): 41-44+108.

[4] Yang Yun, Yang Lingxi, On Application of Linhai Poetry Singing in National Vocal Music Teaching [J]. Journal of Taiyuan University, 2013, 14(3): 105-108. 